Ocula $^{25}$

Occhio semiotico sui media | Semiotic eye on media

www.ocula.it • ISSN 1724-7810 • Vol 22, No 25 (June 2021) • DOI: 10.12977/ocula2021-7

Genere, generi e generazioni

Per una riflessione sociologica sui cambiamenti degli stili di consumo

nel mondo globalizzato: scenari attuali e prospettive future

a cura di Piergiorgio Degli Esposti, Antonella Mascio, Geraldina Roberti

\title{
Il racconto seriale come discorso Rappresentazioni dell'identità di genere in How I met your mother e New Girl
}

\author{
Antonella Capalbi \\ Università di Modena e Reggio Emilia, Modena, IT \\ antonella.capalbi@unimore.it
}

\begin{abstract}
The focus of the article is about the characters of the TV Series since they reflect, by adhesion or by contrast, a series of expectations, professional too, related to gender, with significant repercussions to the audience. The following analysis aims to investigate the representation of male and female characters in two successful TV Series, How I met your mother and New Girl, highlighting the potential impact on the spectator experience.
\end{abstract}

\section{Key Words}

Gender; Work; Representation; Tv Series; Audience

\section{Sommario/Contents}

Introduzione

1. Mondi diretti e mondi indiretti

2. Stereotipi di genere al lavoro

3. How I met your mother

4. New Girl

Conclusioni

Bibliografia 


\section{Ocula ${ }^{25}$}

Vol 22, No 25 (June 2021) • DOI: 10.12977/ocula2021-7

Antonella Capalbi | Il racconto seriale come discorso

\section{Introduzione}

Le costruzioni culturali operate intorno alla percezione della maschilità e della femminilità hanno origini lontane e affondano le radici in un terreno innaffiato con la cultura, seminato con la storia e arato con il costume. L'assegnazione di caratteristiche più immediatamente attribuibili alla maschilità o alla femminilità, riassumibili nella macrodefinzione di stereotipi di genere, tocca diverse sfere del quotidiano, non ultima quella del lavoro, e passa anche per le forme di rappresentazione che all'interno del discorso sociale contribuiscono alla costruzione del genere.

Una certa tipizzazione di genere fa parte anche di un universo, quello della serialità audiovisiva, che trova sempre più spazio all'interno dell'immaginario contemporaneo. Ma quali sono i modelli (maschili e femminili) e le loro caratteristiche, anche professionali, configurati all'interno delle serie televisive statunitensi di successo? E qual è la potenziale ricaduta sull'esperienza spettatoriale? Partendo dall'idea secondo cui il racconto seriale come discorso rappresenta una cucitura tra il mondo diretto (realtà) e il mondo indiretto (serialità), l'analisi che segue si propone di analizzare la tipizzazione di genere di alcuni personaggi, anche in senso professionale, all'interno di serie televisive di successo, in una dialettica che tenta di osservare connessioni e dissonanze tra mondo reale e mondo seriale. Nello specifico, l'analisi sarà incentrata su How I met your mother e New Girl, serie individuate per il grande impatto sul pubblico in termini di costruzione dell'immaginario e poiché caratterizzate da una strutturazione dei personaggi molto differente e a tratti apertamente in contrasto.

\section{Mondi diretti e mondi indiretti}

Se il percorso di riconoscimento della verbalizzazione come attività cognitiva ha ingombrato buona parte della riflessione epistemologica rispetto al linguaggio come perno dei processi cognitivi umani (Chomsky 1981), il cammino di riflessione su quanto anche l'immagine possa costituire un elemento prevalente riguardo alla percezione della realtà è più giovane ma non per questo più acerbo. Stando a Sartori, anzi, l'atto di visione, in quanto atavico e ancestrale, affonderebbe le radici in un tempo molto lontano: ne consegue che il fruitore di esperienze connesse all'immagine «è più un animale vedente che non un animale simbolico. Per lui le cose raffigurate in immagini contano e pesano più delle cose dette in parole. » (Sartori 1999: 14). In sostanza, se è vero che «In ambito scientifico, il costruttivismo radicale e la fenomenologia ci hanno insegnato che noi siamo le parole che ascoltiamo» (Tota 2008: 7), è vero anche che «Nella società contemporanea [...] una delle risorse fondamentali con cui interpretiamo la realtà e con cui diamo significato alla nostra esistenza è costituita dalle immagini. In altri termini, potremmo affermare che noi siamo anche le immagini che vediamo?» (Tota 2008: 7).

Sullo stretto rapporto che intercorre tra il mondo della percezione sensoriale e quello della riproduzione visiva, Eugeni (2013) cerca di fare il punto e 


\section{Ocula ${ }^{25}$}

Vol 22, No 25 (June 2021) • DOI: 10.12977/ocula2021-7

Antonella Capalbi | Il racconto seriale come discorso

analizza la dialettica tra quelli che definisce mondi diretti e mondi indiretti, rilevando quanto l'immaginario risulti largamente influenzato dalle esperienze mediali, anche e soprattutto, come suggerisce Tota (2008), «in contesti come quelli attuali, in cui la natura dell'esperienza si è scissa dalla necessità della compresenza. In altri termini, ciò che vediamo sullo schermo televisivo è in grado di interpellarci in modo profondo e intimo, sino a divenire parte della nostra soggettività» (p. 10). Partendo da questo presupposto, l'esperienza stessa cambia statuto, dal momento che, oltre che fenomeno culturale e relazionale, quando entra in rapporto con il supporto audiovisivo diventa anche un fenomeno seriale, generando comuni aree di percezione e di costruzione del senso (Eugeni 2013): le immagini diventano parte dell'immaginario anche grazie al fatto di poterne fruire nuovamente, affezionarsi a ciò che si vede $\mathrm{e}$ costruire un legame affettivo oltre che simbolico con l'evento mediatico.

La comune esperienza mediale, naturalmente, prescinde dall'idea di sistematizzare dal punto di vista cognitivo quanto osservato in senso univoco e unidirezionale, dal momento che non è detto che «le singole esperienze viventi e vissute dai particolari spettatori siano identiche» (Eugeni 2013: 43) o, per dirla con Tota, non è detto che «il solo fatto di essere esposti all'immaginario sociale ci rende in parte permeabile ai valori, agli atteggiamenti e agli stili di vita che sono quotidianamente rappresentati» (2008: 29). In sostanza, se è vero che la fruizione di esperienze mediali comuni può costituire un tassello importante all'interno del grande mosaico dell'immaginario, è vero anche che vanno sempre considerate le strategie individuali che ogni soggetto diversifica sulla base della propria esperienza culturale e relazionale all'interno del «nuovo regime di finzione» (Augé 1998: 12).

In questo senso, la riflessione sulle proposte di genere all'interno dei prodotti audiovisivi trova spazio, nell'ottica per cui le immagini «rappresentano uno dei terreni fondamentali su cui competere per costruire socialmente le identità di genere, di etnia, di generazione, di classe sociale» (Tota 2008: 21). La rappresentazione, infatti, oltre a essere portatrice di significati culturalmente condizionati e riconosciuti dai fruitori di un determinato prodotto audiovisivo, è parte integrante del processo di costruzione di quegli stessi significati. E se è vero che la costruzione del genere è allo stesso tempo il prodotto e il processo delle sue rappresentazioni (de Lauretiscc 1989), si può dire che la percezione dell'identità di genere non è solo riflessa all'interno delle rappresentazioni audiovisive, ma ne risulta allo stesso tempo forgiata. Per fare un esempio concreto, nell'analisi sociologica delle riviste per teenagers, Chaplin (2007) considera i modi in cui le ideologie influenzano la progettazione delle loro pagine e quindi anche come, «attraverso il materiale visuale, si generano e si spostano i significati nella e attorno alla cultura dei teenagers» (p. 87).

Allo stesso modo le rappresentazioni audiovisive hanno la capacità di veicolare e generare in modo espressivamente potente tutta una serie di significati attorno alla percezione della femminilità e della maschilità, con una ricaduta significativa sull'esperienza spettatoriale. All'interno di quella che significativamente Abercrombie e Longhurst hanno definito "società performativa" (1998), infatti, la costruzione stessa dell’identità individuale risulta 


\section{Ocula ${ }^{25}$}

Vol 22, No 25 (June 2021) • DOI: 10.12977/ocula2021-7

Antonella Capalbi | Il racconto seriale come discorso

essere strettamente connessa con l'esperienza spettatoriale, dal momento che, stando ai due autori, la vita è da considerarsi una performance costante, poiché grazie alle piattaforme social siamo costantemente pubblico e performer allo stesso tempo, all'interno di un contesto mediatico in cui la performance cessa di essere un evento discreto, secondo una lettura che intesse un filo doppio tra la nozione di spettacolarizzazione del mondo e la costruzione narcisistica dell'identità individuale (Scaglioni 2006). In questo senso, non solo siamo le immagini che vediamo (Tota 2008), ma quelle stesse immagini ci definiscono, ci raccontano e alimentano la costruzione della nostra identità. Un fatto estremamente amplificato, quindi, in un contesto come quello contemporaneo caratterizzato da una forte dialettica tra mondo reale e mondo virtuale - costantemente popolato da immagini tratte dalla serialità televisiva - , in cui offline e online convivono, alternandosi, in quello che Dean (2010) definisce opportunamente "blogipelago", e non blogosphere, per sottolineare gli spazi di reciproca connessione e separazione tra le due sfere, in un gioco di identità forgiate dal virtuale, che diventano appendici, e non antagoniste, di quelle costruite nella vita reale (Floridi 2014).

\section{Stereotipi di genere al lavoro}

Muovendo dai presupposti precedentemente illustrati, dunque, si può osservare come una certa rappresentazione dei personaggi maschili e femminili e delle loro caratteristiche, anche professionali, all'interno dell'universo seriale può avere una ricaduta sull'esperienza spettatoriale.

Stando a OECD (2017b), per esempio, la tipizzazione stereotipata delle professioni ha una capacità di influenzare le scelte professionali più delle reali abilità del singolo individuo in un determinato settore. In questo senso risulta molto interessante osservare in che modo la tipizzazione di genere delle professioni è veicolata all'interno della serialità televisiva, la cui fruizione ruota attorno alla grande categoria del target giovanile, caratterizzata dal delicato processo di formazione del sé.

Le implicazioni da un punto di vista lavorativo sono evidenti nella misura in cui una determinata percezione del sé può influenzare le scelte di formazione alla base dei futuri destini professionali. E infatti, stando ancora a OECD, «Disparities between the interests and preferences of 15-year-old boys and girls, and their academic strenghts, and in their participation in after-school courses or training activities may therefore have a significant impact on gender gaps later on» (2017b: 107). Determinate influenze socio-culturali sulle scelte formative avrebbero, quindi, una ricaduta concreta in ambito professionale rispetto al gender gap di un determinato settore. In questo senso, dunque, prima di addentrarsi nell'analisi delle serie individuate, vale la pena di riprendere l'analisi di Wilk (2007) relativa alla costruzione degli stereotipi di genere e al loro impatto sulle scelte professionali degli individui. Stando all'autore, a seguito della rivoluzione industriale e alla conseguente esternalizzazione di tutte le attività produttive al di fuori della casa, luogo femminile per eccellenza sin dalla Grecia antica (Cantarella 1981), la costruzione di un certo dualismo 


\section{Ocula ${ }^{25}$}

Vol 22, No 25 (June 2021) • DOI: 10.12977/ocula2021-7

Antonella Capalbi | Il racconto seriale come discorso

di genere nella percezione del lavoro fu conseguenza necessaria, secondo un binomio oppositivo che vedeva assegnate alle attività svolte al di fuori della casa (dall'uomo) caratteristiche legate al ragionamento logico-razionale e, per contrasto, alle attività svolte in casa (dalla donna) quelle legate alla sfera del privato e quindi dell'emotività:

pubblico/privato

economia/famiglia

uomo/donna

razionale/emozionale

mente/corpo

storico/naturale

oggettivo/soggettivo

scienza/umanità

scienza economica/sociologica

competitivo/educativo

indipendente/dipendente

(Wilk 2007: 37)

Questa costruzione di tipo binomiale in senso culturale, riassumibile nella macrocategoria degli stereotipi di genere, ha di fatto delle ricadute sulle aspettative che investono uomini e donne, nonché sulle scelte di formazione e poi professionali. Stando a Michelini, un effetto concreto di questa concezione oppositiva tra il dentro e il fuori si è tradotto a livello storico nel recepire il lavoro maschile come piu connesso al concetto di produzione (all'esterno) e il lavoro femminile piu connesso al concetto di riproduzione (all'interno). $\mathrm{Ne}$ deriva la dicotomia oppositiva che segue:

\section{Lavoro riproduttivo}

Non retribuito

Isolamento

Senza controllo

Relazioni affettive

Non considerato come lavoro

Ripetitivo e invisibile

Senza contributi previdenziali

(Michelini 2002:254)

\section{Lavoro produttivo}

Produttivo

Relazioni sociali

Condizioni lavorative

Relazioni professionali

Riconosciuto socialmente

Possibilità di avanzamento di carriera

Contributi previdenziali

In questo senso, il lavoro femminile risulta immediatamente associato alla dimensione dell'interno, motivo per cui, stando a uno dei tre principi tracciati da Bourdieu a proposito del dominio maschile (1998), le professioni femminili risultano essere molto spesso prolungamento delle funzioni domestiche (insegnamento, assistenza, servizi) e quindi, in quanto tali, spesso percepite come accessorie (Fontana 2002) e slegate da contesti in cui sono richieste attitudini logico-razionali. Non a caso, «In higher education, young women are under-represented in the fields of science, technology, engineering and 


\section{Ocula ${ }^{25}$}

Vol 22, No 25 (June 2021) • DOI: 10.12977/ocula2021-7

Antonella Capalbi | Il racconto seriale come discorso

mathematichs (STEM). Women account for less than $20 \%$ of entrants into tertiary-level computer science programmes in OECD countries are only around $18 \%$ of engineering entrants» (OECD 2017b: 105). Questa esigua presenza in termini percentuali all'interno del settore sopracitato si interseca con scelte di tipo educativo foraggiate anche da fattori di carattere socio-culturale, in cui rientra senz'altro un'influenza da parte dei modelli proposti all'interno dei media: «people live purposeful lives, but socialisation actors - family, school, peers, the media - play a major role» (Addis et al. 2012:72). Muovendo da questi presupposti, come precedentemente annunciato, l'analisi che segue si proporrà di indagare la strutturazione di modelli maschili e femminili all'interno di due serie televisive di successo, How I met your mother e New Girl, rilevando l'influenza degli stereotipi di genere sulla costruzione dei personaggi e delle loro caratteristiche, anche professionali.

\section{How I met your mother}

Ideata da Craig Thomas e Carter Bays, nel 2005 la serie di culto How I met your mother faceva il suo ingresso nel palinsesto della CBS e avrebbe fatto compagnia a una grossa fetta di pubblico per circa dieci anni. Partendo da un'impostazione che si sviluppa in senso diacronico, proprio perché al contrario dei serial anni Cinquanta non risulta «suddivisa in capitoli, cioè segmenti narrativi autoconclusivi senza sviluppo cronologico delle vicende» (Grasso e Scaglioni 2003: 124), la serie sviluppa un racconto che parte dalla fine e che orienta tutta la narrazione intorno a un grande flashback.

Nella prima puntata, il protagonista Ted Mosby avvia la lunga narrazione con lo stratagemma del racconto ai propri figli su come ha conosciuto la loro madre. I due personaggi rappresentano l'artificio narrativo con cui cristallizzare il pubblico, ugualmente in ascolto e seduto sullo stesso divano dei due figli desiderosi di conoscere l'epilogo della vicenda. Un epilogo che conoscerà varie dilatazioni temporali e diverse digressioni che permettono ai registi di approfondire la storia e le vicende non solo del protagonista Ted, ma anche dei suoi compagni di viaggio Lily, Marshall, Robin e Barney. Il gruppo di amici diventa il fulcro attorno a cui si arrotano le vicende più quotidiane nella New York tanto amata dal protagonista, secondo un affresco narrativo realistico che vede invecchiare, cambiare e modificare gli attori protagonisti insieme ai personaggi della serie, a dimostrazione di come «Se prima il confine tra una serie (a episodi autoconclusivi) e un serial (con una storia continuativa) era netto, oggi questi territori sfumano l'uno nell'altro» (Mittel 2017: 14). I personaggi di How I met your mother esemplificano perfettamente il concetto di mimesi che sta alla base della narrazione seriale e che induce un profondo senso di identificazione da parte del pubblico con le vicende narrate (Grasso e Scaglioni 2003). L'andamento cronologico delle puntate, e l'approfondimento della caratterizzazione dei personaggi in senso diacronico, infatti, permettono al pubblico di scegliere i propri protagonisti, identificarsi, crescere con loro. In questo senso, i loro modi di dire, le frasi idiomatiche, i tormentoni verbali diventano parte del quotidiano e costituiscono moneta sonante all'interno 


\section{Ocula ${ }^{25}$}

Vol 22, No 25 (June 2021) • DOI: 10.12977/ocula2021-7

Antonella Capalbi | Il racconto seriale come discorso

del sistema di scambi linguistici nel reale. Esemplificativo è il caso di Barney Stinson, icona del ricco, cinico e patriottico playboy statunitense, sempre in abito a sottolineare il suo status privilegiato (Seibert Desjarlais 2018), il cui tormentone, «legen... wait for... dary!», fa da filo conduttore anche alle tante fanpage costruitesi intorno al personaggio. Un fatto estremamente significativo per questa analisi, dal momento che «The representation of masculine characters and the ways in which masculinity is enacted within a television show have the potential to perpetuate discourse that reasserts the power of hegemonic masculinity» (Seibert Desjarlais 2018: 171). Come gli altri personaggi, anche quello di Barney conoscerà un cambiamento e una certa modifica dei suoi comportamenti, soprattutto in virtù dell'amore sincero provato per Robin, co-protagonista della serie e amore indimenticato di Ted. Il personaggio di Robin costituirà un motore narrativo forte nelle interazioni con i due personaggi costruite all'interno delle diverse puntate nel solco di inseguimenti, rotture, riavvicinamenti e relazioni tormentate, bilanciate dalla solidità relazionale rappresentata invece dai due personaggi di Lily e Marhsall, fidanzati dalla prima puntata, poi marito e moglie e infine genitori. Proprio il personaggio di Robin permette di addentrarci nel tema oggetto della trattazione, poiché costituisce un personaggio femminile a tratti controverso. Indipendente, ironica, forte e carismatica, Robin risulta essere il naturale oggetto d'attenzione per i due personaggi maschili principali, ma allo stesso tempo è la causa delle contraddizioni interiori vissute da Ted a causa del proprio mancato desiderio di maternità. La volontà del personaggio di non avere figli in virtù del suo desiderio di carriera nel mondo del giornalismo rappresenta una canonizzazione dello stereotipo più classico della donna indipendente che, in virtù della carriera, tradisce le aspettative di genere che ruotano attorno alla sua femminilità e, quindi, al suo ruolo riproduttivo.

$\mathrm{E}$, infatti, la costruzione stessa del personaggio di Robin si struttura attorno a questa indicazione narrativa: come è sottolineato in più punti della serie, Robin non solo non vuole avere figli come ogni donna dovrebbe volere ma, all'opposto, beve come un uomo, si diverte come un uomo, spara come un uomo - nonostante sia canadese! - e, come un uomo, investe buona parte del suo tempo e delle sue aspirazioni nella carriera (Seibert Desjarlais 2018). Questa scelta di vita trova un momento di catarsi quando curiosamente Robin capisce non solo di non desiderare figli, ma anche di non poterne avere per un problema di tipo biologico (S7E12). È interessante osservare come, nella narrazione costruita attorno al personaggio, da sempre caratterizzato dalla sicurezza di non volere figli, si crei una svolta nel momento in cui la non-maternità passa dall'essere una scelta all'essere una costrizione. Un fotogramma in cui beve da sola del caffè su una panchina innevata sintetizza il momento di impasse emotivo vissuto dalla giovane donna, che nel corso della puntata svela un lato sensibile mostrato a fatica e lo svela proprio in relazione alla definitiva impossibilità di diventare madre. La tipizzazione di genere corre sul filo intessuto dalle caratteristiche che socialmente sono assegnate alla femminilità, tra cui necessariamente il desiderio di essere madre. Sembra quasi che i creatori della serie in questa fase tentino di riabilitare il personaggio di Ro- 


\section{Ocula ${ }^{25}$}

Vol 22, No 25 (June 2021) • DOI: 10.12977/ocula2021-7

Antonella Capalbi | Il racconto seriale come discorso

bin, e con lei la sua femminilità, legittimando il suo rifiuto nei confronti della maternità, e quindi del suo "lavoro" riproduttivo, con una disfunzione di tipo biologico, non più percepita dal pubblico come scelta desiderata e consapevole, dimostrando quindi una certa persistenza degli stereotipi di genere nella costruzione del personaggio.

$\mathrm{Al}$ di là di questo episodio specifico, inoltre, è interessante osservare come la figura di un personaggio femminile indipendente venga incarnata da una serie di velleità tipicamente e socialmente connotate come maschili, quali il desiderio di carriera a scapito della famiglia. In questo senso, nel gioco di specchi dal punto di vista del genere costruito nella serie, all'opposto, il personaggio di Ted incamera una serie di prerogative percepite socialmente come femminili e assegnate a quella che Gill definisce la nuova tipologia di uomo rappresentata nella cultura popolare occidentale: «sensitive, emotionally aware, respectful of women, and egalitarian in outlook» (2003: 37). Come non si manca di sottolineare altrettanto spesso, Ted cucina come una donna, è sensibile come una donna, si innamora facilmente come una donna, è ossessionato dall'idea di sposarsi come una donna (Thompson 2015: 32,) e, in ultima analisi, ha anche un lavoro da donna. Il protagonista, infatti, è un architetto e, come risulta evidente in un momento di euforia forsennata in cui lui e Barney immaginano di adottare insieme un bambino (So7E11), la percezione del lavoro di architetto viene esplicitata da Barney, impiegato in un settore altamente male-dominated come quello finanziario, che nella finta strutturazione dei ruoli genitoriali rappresenterebbe il padre stupito dalla scelta di Ted - la madre - di continuare a lavorare anche dopo aver adottato il bambino:

- Quindi tu vuoi tenere il tuo posto di lavoro?

- Cosa? Certo che terrò il posto, perché mai dovrei lasciarlo?

- Beh... Insomma, questa faccenda dell'architettura in fondo in fondo non è solo... un hobby?»

La chiosa sarcastica del maschilista Barney durante la lite con l'amico mascherata da discussione tra coniugi, in aderenza al ribaltamento comico costruito nell'episodio, assembla tutta la narrazione sociale attorno all' idea, radicata a lungo nel senso comune, secondo cui il lavoro femminile, in quanto accessorio, possa e debba essere sottomesso alle esigenze di famiglia, in modo da non compromettere la «funzione essenziale» (Fontana 2002: 18), quella domestica e riproduttiva. E, in effetti, questa rappresentazione dicotomica del lavoro maschile e femminile è ben documentata nella relazione tra Marshall e Lily, insegnante d'asilo lei e avvocato in una grossa azienda lui. Per quanto anche le vicende professionali di Lily e Marshall subiscano modifiche nel corso delle puntate, nella strutturazione dei ruoli all'interno della coppia-modello della serie si osserva una forte impostazione del primo dei tre principi tracciati da Bourdieu (1998) a proposito della divisione sessuale del lavoro, secondo cui, come precedentemente espresso, le professioni femminili risultano essere prolungamento delle funzioni domestiche (insegnamento, assistenza, servi- 


\section{Ocula ${ }^{25}$}

Vol 22, No 25 (June 2021) • DOI: 10.12977/ocula2021-7

Antonella Capalbi | Il racconto seriale come discorso

zi). Da questo punto di vista, Lily rappresenta la perfetta canonizzazione del modello lavorativo femminile, in cui rientra in parte anche la professione di Robin nel settore della Comunicazione, per quanto nel suo caso lo strappo alla tipizzazione di genere sia costituito dal desiderio di immolare le proprie scelte in campo affettivo in favore della carriera. Se Lily è una donna, Robin lo è un po' meno proprio in virtù di questa scelta. Non essendo né moglie, a esclusione dell'ultima stagione, né madre, per tutta la durata della serie, Robin dunque viene presentata come un personaggio femminile solo dal punto di vista della seduzione e maschile dal punto di vista delle scelte di vita, pur vivendo aspettative sociali legate al genere che, oltre al tema della maternità, investono anche la sfera della condotta sessuale. Se Lily, prima fidanzata, poi moglie e infine madre, non ha altro partner all'infuori di Marshall nel corso della serie, il personaggio di Robin vive una serie di flirt e avventure di una notte. Piuttosto esemplificativa risulta essere quella che i protagonisti ipotizzano abbia vissuto ad Halloween e, nello specifico, risulta interessante per il modo in cui i personaggi pensano di averla scoperta. Come viene specificato all'inizio dell'episodio (S7E6), è tradizione per i tre personaggi maschili, infatti, appostarsi sulle scale dell'appartamento di Ted il giorno dopo la notte che precede Ognissanti per osservare la vera parata di Halloween: quella della vergogna da parte delle ragazze che, imbarazzate, tornano a casa di mattina presto dopo una notte di bagordi, indossando ancora i costumi della sera prima. ̇̀ in questo contesto che vedono comparire Robin, vestita da infermiera sexy, di ritorno in realtà dalla partecipazione come comparsa a uno spot pubblicitario di bassa qualità, come si scoprirà a fine puntata. $\mathrm{Al}$ di là dell'epilogo della vicenda, risulta significativo osservare come i tre personaggi maschili, ma soprattutto il romantico Ted e il saggio Marshall, accomunati dal fatto di essere bianchi, eterosessuali e professionisti della classe medio-alta (Seibert Desjarlais 2018), si trovino a costruire un club temporaneo di soli uomini intorno allo sberleffo di una scelta sessuale libera da parte delle giovani donne di New York, prede sistematiche di Barney, unico da cui a livello narrativo ci si aspetterebbe questo comportamento, confermando quanto espresso da Seibert Desjarlais (2018) su come l'egemonia maschile viene incorporata ed esercitata anche inconsciamente.

Il temporaneo club, infatti, si struttura sulla base di un sodalizio maschile a suon di catcalling in forma di commenti sarcastici rivolti alle ragazze e risulta costruito sull'idea per cui una donna che vive in maniera libera la propria sessualità, non sottomettendola alla funzione riproduttiva, costituisce oggetto di derisione. In linea di contrasto rispetto a questa concezione, secondo un dialogo aperto proprio con questa puntata, si pone un altro grande successo seriale degli ultimi anni: New Girl (2011-2018).

\section{New Girl}

Prodotta e interpretata da Zooey Deschanel, e ideata da Elisabeth Meriwether, la serie, pur facendo specifico riferimento ai grandi antecedenti all'interno del genere, in alcuni punti sembra porsi in aperto contrasto con 


\section{Ocula ${ }^{25}$}

Vol 22, No 25 (June 2021) • DOI: 10.12977/ocula2021-7

Antonella Capalbi | Il racconto seriale come discorso

How I met your mother. All'interno di una girandola di vicende ambientate a Los Angeles, la protagonista Jess, dopo la rottura di una relazione importante, inizia una convivenza con i tre coinquilini Nick, Winston e Schmidt, che imparano ad amarne le stranezze. Attorno alle avventure urbane dei quattro protagonisti, e a quelle di personaggi collaterali significativi come Cece, la migliore amica di Jess, e Coach, coinquilino del loft a fasi alterne, ruota lo schema narrativo della serie. Il personaggio di Jess, "adorkable" nella sua stramberia - il termine è stato coniato per identificare il personaggio fondendo i termini "adorable" (adorabile) e "dork" (socialmente inetta) (McIntyre 2015) -, sembra essere una risposta allo stereotipo di genere che vede una donna emancipata in guerra con la propria femminilità. Divertente ma severa, stravagante ma stacanovista, la protagonista della serie, per quanto nella lettura di McIntyre (2015) incameri alcune punte di conservatorismo, da diversi punti di vista risulta essere una provocazione ragionata alle caratteristiche comunemente assegnate alla femminilità e, di contro, alla maschilità. L'intera serie, e la strutturazione dei personaggi, sembra rispondere a questa esigenza. E lo dimostra il dialogo programmatico tra $\mathrm{S}_{\mathrm{EE}} 7 \mathrm{di}$ How I met your mother, quella relativa alla parata della vergogna di Halloween, e S4E18 di New Girl, in cui il modello è ripreso in senso conflittuale a partire dal titolo della puntata, che risulta essere significativamente proprio "La sfilata della vergogna". Siamo nuovamente a una festa di Halloween, stavolta nella Los Angeles sede d'ambientazione della serie, e le due amiche Jess e Cece, dopo aver bevuto un bicchiere di troppo, si ritrovano il mattino dopo a casa dei due uomini con cui hanno passato la notte, pronte a rincasare in abito da sera e tacchi, secondo una vera «sfilata della vergogna, muoviamoci!», - suggerisce proprio il personaggio di Cece, strizzando evidentemente l'occhio all'antecedente narrazione seriale che ha utilizzato questa definizione. Secondo una strutturazione che fa programmaticamente riferimento ad How I met your mother, quindi, New Girl prova a leggere il tema dell'imbarazzo sociale relativo a una condotta sessuale libera dal punto di vista femminile. Ed è in questo senso che si crea un profondo strappo rispetto allo sguardo maschile proposto da How I met your mother, perché nel corso della puntata Jess suggerisce che non c'è proprio niente di cui vergognarsi nell'aver passato la notte fuori e che, se una sfilata deve verificarsi in tacchi alti e trucco sbavato, dev'essere dell'orgoglio per la propria condotta consapevole e libera, all'interno di una puntata in cui, ancora secondo un dialogo aperto con How I met your mother, si scoprirà che anche le due donne, proprio come Robin, in realtà non hanno avuto alcuna avventura serale, ma si sentono di rivendicarla anche solo in potenza in quanto atto dovuto alla loro femminilità. Secondo poi il classico andamento narrativo scanzonato, lungo il tragitto le due donne coinvolgeranno casualmente anche un ex partner di Jess, in imbarazzanti abiti da clown in occasione di una festa per bambini, invitato ad aggregarsi alla sfilata come monito al non doversi vergognare per aver perso il lavoro di insegnante ed essere obbligato a guadagnare intrattenendo feste di compleanni. La sfilata della vergogna di How I met your mother, commentata a suon di sberleffi dal club temporaneo di soli uomini formatosi sulle scale 


\section{Ocula ${ }^{25}$}

Vol 22, No 25 (June 2021) • DOI: 10.12977/ocula2021-7

Antonella Capalbi | Il racconto seriale come discorso

dell'appartamento di Ted, diventa in New Girl un sodalizio umano, non solo al femminile, per invitare a liberarsi di alcune catene invisibili che attorniano la vita, sessuale e non, di uomini e donne. Questo dialogo aperto con la serie How I met your mother, posto in senso assolutamente ironico, rende lo spaccato della diversa impostazione di New Girl. Una diversa impostazione che è rintracciabile anche nella strutturazione dei ruoli professionali all'interno delle puntate. In antilogia con il modello rappresentato da Robin della donna di carattere e in carriera, con spiccati comportamenti connotati socialmente come maschili, non solo Jess è una maestra, professione altamente tipizzata come femminile, ma è un personaggio che rivendica ogni traccia di ciò che è socialmente tipizzato come femminile anche da un punto di vista del look. Ballerine, vestitini alla francese e fiocchi di tutti i colori adornano il personaggio, che però tiene a rivendicare la sua emancipazione in più punti della serie. Proponendo un modello di autonomia e indipendenza che non passa per la necessaria frustrazione della propria femminilità, la maestra Jess, poi preside, si dimostra seria e impegnata sul lavoro, pur amando le borsette, perché «non significa che non sono femminista! Io sono una femminista che ama le borsette. Dove altro dovrei tenere i miei scritti femministi? In una borsetta, ecco dove!», suggerisce ironicamente il personaggio stesso (S4E10). L'importanza intorno alla dimensione professionale del personaggio di Jess è dimostrata dal fatto che in molte puntate ne diventa motore narrativo: a partire dalla serie di episodi in cui perde il lavoro arrivando a quelle in cui corona il sogno di diventare preside, sfondando il soffitto di cristallo che vede da sempre le professioni apicali precluse alle donne (Addis 1997).

La strutturazione di genere proposta in New Girl, quindi, scombina le carte e offre una proposta delle professioni in senso critico e originale, non solo dal punto di vista femminile ma anche da quello maschile. È il caso di Schmidt, dongiovanni del loft, che lavora in un settore tipizzato come femminile, quello del Marketing e della Comunicazione. Nel corso delle puntate, si descrivono le difficoltà del personaggio nel rapportarsi a un contesto composto da sole donne, proponendo una riflessione su come il paradigma della tipizzazione professionale non investa solo il lato femminile della società, ma anche quello maschile per converso. Stando a OECD (2017b), per esempio, nel 2015 solo il $3 \%$ degli studenti maschi ha dichiarato di voler intraprendere la carriera da insegnante, a fronte dell' $8 \%$ di studentesse. Un fatto estremamente correlato con la tipizzazione femminile della professione nell'immaginario, dal momento che «the perceived feminisation of the teaching profession may be a factor in teenage boys' low motivation and lack of involvement in school» (OECD 2017b: 123). In generale l'attenzione alla ricaduta degli stereotipi di genere non solo sul lato femminile della società ma anche su quello maschile sembra fare da filo conduttore a diverse puntate della serie, a partire da un episodio (S4E8) in cui i coinquilini del loft, rimasti soli a casa senza Jess, si danno a una «serata tra uomini!», durante la quale, seppur partiti con l'intenzione di passarla tra birre e aneddoti virili, finiscono a confidarsi le proprie emozioni più profonde e a smascherare il proprio lato sensibile. Secondo un model- 


\section{Dcula ${ }^{25}$}

Vol 22, No 25 (June 2021) • DOI: 10.12977/ocula2021-7

Antonella Capalbi | Il racconto seriale come discorso

lo che sembra invitare il pubblico maschile a non ritenere l'espressione della propria emotività un fatto lontano da sé solo perché socialmente tipizzato come femminile - razionale vs emozionale (Wilk 2007) - , la serie dà vita poi a un ribaltamento ironico dei più divertenti ( $\left.\mathrm{S}_{5} \mathrm{E} 17\right)$, quando Schmidt cerca di dare prova (non richiesta) della sua virilità alla fidanzata Cece, inducendo i suoi compagni di viaggio a ingaggiare una rissa con dei brutti ceffi incontrati durante il viaggio per il suo addio al celibato. Dopo una generale riluttanza da parte di tutti, il confronto corpo a corpo prende vita sulle note ironiche di Roar cantata da Katy Perry il cui ritornello, "I have the eye of the tiger", strizza programmaticamente l'occhio alla colonna sonora di Rocky, secondo un ribaltamento della lotta che non solo a livello sonoro risulta distante da ogni prototipo di mascolinità bruta. Dopo l'inevitabile sconfitta dei protagonisti, il personaggio di Nick rincuora l'amico sul fatto di non dover temere di essere poco virile per Cece, perché lo è già secondo una definizione contemporanea di mascolinità nel momento in cui dimostra quotidianamente di essere sinceramente innamorato di lei. $\mathrm{Al}$ di là del lieto fine canonico, il dialogo tra $\mathrm{i}$ due uomini svecchia in modo incredibilmente leggero il ruolo dell'uomo cacciatore e della donna preda che va protetta, infilando con destrezza nel mare magnum dell'immaginario un concetto importante che parla di aspettative di genere non solo al femminile ma anche al maschile. In poche battute, il binomio oppositivo degli stereotipi di genere tracciato da Wilk (2007) viene ribaltato, suggerendo in forma ironica una versione rinnovata della percezione di sè anche relativamente alla parte maschile della società e rivelando una strutturazione di genere differente, anche e non solo di tipo professionale, rispetto a quella proposta all'interno di How I met your mother.

\section{Conclusioni}

L'analisi effettuata ha permesso di osservare quanto la rappresentazione audiovisiva di tipo seriale abbia una forte presenza nell'immaginario e una potenziale ricaduta rispetto alla costruzione del sé, anche da un punto di vista professionale. Partendo dagli esempi forniti all'interno delle serie televisive oggetto di questa analisi, si è potuto osservare come una certa tipizzazione di genere, anche delle professioni, costruita su base culturale ma spesso percepita come ontologica, possa risultare cementata anche dalla dialettica della rappresentazione. Nello specifico, nelle serie analizzate sembra essere confermata, per adesione (How I met your mother) o per contrasto e in senso critico (New Girl), una certa persistenza degli stereotipi di genere, anche in ambito lavorativo, caratterizzata dai poli oppositivi tracciati da Wilk (2007) e Michelini (2012).

Si è potuto evidenziare, inoltre, che l'influenza degli stereotipi di genere nella costruzione dei personaggi e delle loro caratteristiche, anche professionali, risulta essere madre di possibili ricadute sull'esperienza spettatoriale in termini di percezione del sé.

In questo senso, riprendendo il concetto di inquinamento visuale (Tota 2008) e di società performativa (Abercrombie, Longhurst 1998), si conferma 


\section{Ocula ${ }^{25}$}

Vol 22, No 25 (June 2021) • DOI: 10.12977/ocula2021-7

Antonella Capalbi | Il racconto seriale come discorso

una forte connessione tra serialità e pubblico, nel solco di una costruzione dell'identità di genere, anche professionale, che attraversa il terreno della rappresentazione audiovisiva.

\section{Bibliografia}

Abercrombie, Nicholas; Longhurst, Brian

1998 Audiences. A Sociological Theory of Performance and Imagination, London, Sage.

Addis, Elisabetta

1997 Economia e differenza di genere, Bologna, Clueb.

Addis, Elisabetta et al.

2012 Meta-analysis of gender and science research. Synthesis report, Luxembourg, European Union.

Augé, Marc

1997 La guerre des rêves, Paris, Seuil; (tr. it. La guerra dei sogni, Milano, Elèuthera, 1998).

Bourdieu, Pierre

1998 La domination masculine, Paris, Seuil; (tr. it. Il dominio maschile, Milano, Feltrinelli).

Cantarella, Eva

1981 L'ambiguo malanno: condizione e immagine della donna nell'antichità greca e romana, Roma, Editori Riuniti.

Chaplin, Elizabeth

2007 Cultural Studies e rappresentazioni visuali: la creazione dei significati attraverso gli aspetti visuali del testo, in Harper, D. e Faccioli, P. (a cura di), Mondi da vedere. Verso una sociologia più visuale, Milano, Franco Angeli.

Chomsky, Noam

1975 Reflections on language, London, Temple Smith; (tr. it. Riflessioni sul linguaggio, Torino, Einaudi, 1981).

de Lauretis, Teresa

1989 Technologies of gender. Essays on Theory, Film, and Fiction, Macmillan, Houndmills.

Dean, Jodi

2010 Blog Theory: Feedback and Capture in the Circuits of Drive, Cambridge, Polity Press.

Eugeni, Ruggero

2013 Semiotica dei media. Le forme dell'esperienza, Roma, Carocci.

Floridi, Luciano

2014 The Onlife Manifesto. Being Human in a Hyperconnected Era, New York, Springer Open. 


\section{Ocula ${ }^{25}$}

Vol 22, No 25 (June 2021) • DOI: 10.12977/ocula2021-7

Antonella Capalbi | Il racconto seriale come discorso

Fontana, Renato

2002 Il lavoro di genere. Le donne tra vecchia e nuova economia, Roma, Carocci.

Gill, Rosalind

2003 "Power and the Production of Subjects: A Genealogy of the New Man and the New Lad", The Sociological Review, 51, pp. 34-56.

Grasso, Aldo; Scaglioni, Massimo

2003 Che cos'è la televisione. Il piccolo schermo fra cultura e società: $i$ generi, l'industria, il pubblico, Milano, Garzanti.

McIntyre, Anthony P.

2015 "Isn't She Adorkable! Cuteness in the Star Text of Zooey Deschanel", Television \& New Media, 16(5), pp. 422-438.

Michelini, Cristina

2002 Gli stereotipi di genere e il mondo del lavoro, in Moschini L. (a cura di), Gli stereotipi di genere. Dalla comunicazione mediatica al mondo del lavoro, Roma, Aracne.

Mittel, Jason

2015 Complex TV: the poetics of contemporary television storytelling, New York, New York University Press; (tr. it. Complex tv. Teoria e tecnica dello storytelling delle serie tv, Roma, Minimum Fax, 2017).

$\mathrm{OECD}$

2017 The Pursuit of Gender Equality: An Uphill Battle, Parigi, Publishing.

Sartori, Giovanni

1999 Homo videns, Roma-Bari, Laterza.

Scaglioni, Massimo

2006 Tv di culto: la serialità televisiva americana e il suo fandom, Milano, V\&P.

Seibert Desjarlais, Stevie, K.

2018 "How I Met Your Masculinity: Contrasting Male Personas Portrayed on How I Met Your Mother", Journal of Popular Film and Television, 46(3), pp. 169178 .

Thompson, Lauren Jade

2015 "Nothing Suits me Like a Suit: Performing Masculinity in How I Met Your Mother", Critical Studies in Television, 10(2), pp. 21-36.

Tota, Anna Lisa (a cura di)

2008 Gender e media. Verso un immaginario sostenibile, Roma, Meltemi.

Tota, Anna Lisa

2008 Inquinamento visuale e sostenibilità dell’immaginario, in Tota, A. L. (a cura di), Gender e media. Verso un immaginario sostenibile, Roma, Meltemi, pp. $15-38$.

Wilk, Richard

1996 Economies and cultures: foundations of economic anthropology, Boulder, Westview Press; (tr. it. Economie e culture. Introduzione all'antropologia economica, Milano, Mondadori, 2007). 


\section{Dcula ${ }^{25}$}

Vol 22, No 25 (June 2021) • DOI: 10.12977/ocula2021-7

Antonella Capalbi | Il racconto seriale come discorso

Antonella Capalbi ha conseguito un dottorato di ricerca presso l'Università di Modena e Reggio Emilia con una tesi di ambito visuale. Tra le sue recenti pubblicazioni: "Faccio dunque sono? Trasformazioni del lavoro e dell'identità di genere nelle rappresentazioni audiovisive di Short on Work" (con G. Piscitelli), in Sociologia del Lavoro, Milano, FrancoAngeli, 2020; “'Short on Work': rappresentazioni audiovisive tra ricerca, lavoro e vita quotidiana” (con Fabbri T. M., Costantini E., Piscitelli G., Risso G. L. ), in Sociologie visuelle et filmique: Le point de vue dans la vie quotidienne, 2018, Genova, Genova University Press; "Millennium e Cinquanta sfumature. Modelli di relazione tra letteratura e media", in Rossi E. (2018) (a cura di), Senza di me non vali niente. La violenza verbale, emotiva e psicologica nelle relazioni intime, Roma, Aracne.

Giornalista pubblicista, attualmente è co-curatrice di Short on Work, concorso internazionale di cortometraggi sul tema del lavoro ideato e prodotto dalla Fondazione Marco Biagi, e assegnista di ricerca presso Unimore. Parallelamente all'attività di ricerca ha esordito nella narrativa nel 2017 pubblicando una raccolta di racconti dal titolo Storie di vita al 30\%, Palermo, Leima. Nel 2020 ha collaborato alla raccolta di racconti dal titolo Futuro Anteriore, Bari, Gelsorosso, a cura di Michele Marolla. 


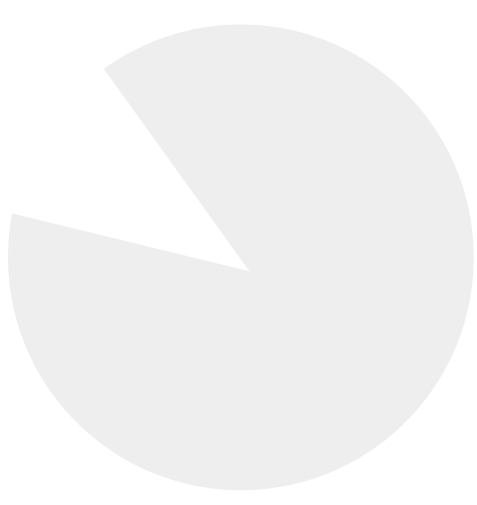

